\title{
Measurement strategy to study radon source, entry and dilution rates in energy-efficient buildings in Russia
}

\author{
Aleksey Vasilyev $^{1 \mathrm{a}}$, Ilia Yarmoshenko ${ }^{1}$, and Michael Zhukovsky ${ }^{1}$ \\ ${ }^{1}$ Institute of Industrial Ecology UB RAS, 620990, Yekaterinburg, Russia
}

\begin{abstract}
Main physical quantities, which control indoor radon in modern energy-efficient buildings of Russia was studied basing on the results of radon survey in Ekaterinburg, Russia. It was approved that measures to increase energy efficiency lead to reduction in the ventilation rate and high accumulation of radon concentrations indoors. Long-term measurements with application of proposed mathematical methods give an improved understanding of the radon entry processes. Combined use of integrative and time series (differential) measurements provides effective way to develop standard protocol for radon measurements in energy-efficient multi-storey buildings.
\end{abstract}

\section{Introduction}

In this study, attention will be confined to the problem of population exposure to radon in context of energy efficient construction technologies. With the introduction of highperformance insulating materials and other architectural and construction concepts air permeability of building envelope becomes sharply reduced. Besides effective reduction of air permeability of building envelope, conditions for increase of many indoor-generated air pollutants are created $[1,2]$. Radon is the one of the most important indoor pollutant from the standpoint of radiation safety. Therefore, the need to consider the protection of public health from radon exposure in conditions of energy-efficient construction is a major task in the field of radiation hygiene.

Multi-storey block buildings represent the main type of dwellings in modern cities of Russia. There are two important sources that contribute to indoor radon: exhalation from the building materials and from the soil (Figure 1). Buildings materials are supposed to be dominant radon source, as proved by continuous measurements of radon concentration in multi-storey dwellings with typical natural ventilation system [3]. Air exchange rate (AER) between outdoor and indoor atmosphere has significant effect on the radon concentration, but implementation of energy-efficient measures result in an increase of air permeability of building envelope. Conditions for high indoor radon concentrations are created.

\footnotetext{
${ }^{a}$ Corresponding author: vav@ecko.uran.ru
} 


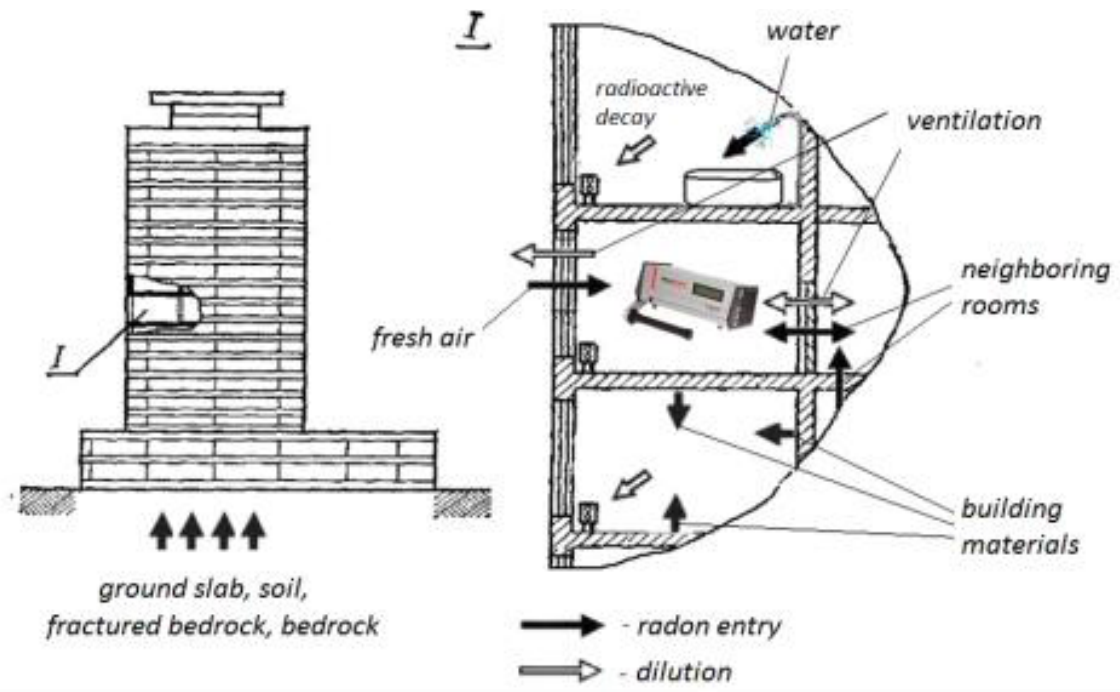

Fig. 1. Indoor radon in multi-storey building: parameters of interest.

The main task of the study is estimation of the main physical quantities, which control indoor radon in modern energy-efficient buildings of Russia, such as properties of radon source, entry and dilution rates.

\section{Materials and methods}

The sample of dwellings for analyses includes 11 multi-storey buildings having been constructed after 2000 with implementation of the features of energy-efficient construction in Ekaterinburg, Russia. The building characteristics are the following: monolithic reinforced concrete structures, high-performance windows and exterior heat insulating walls of aerated concrete.

A method, that is based on high-resolution radon time series and allows to determine the ventilation rate corresponding inactive and active modes of room use of radon entry analysis, was developed earlier [4]. The method consists of the continuous measurements of radon concentration, temperature and pressure difference between indoor and outdoor atmosphere and analysis. Dwellings were equipped with AlphaGUARD monitors. Measurement interval was set to 60 minutes. Duration of measurements was 6 months. The beginning of the measurements was assigned on winter or summer, so duration of measurements covered cold and warm seasons.

A typical form of radon concentration time series is shown in Figure 2 (upper part). Points are results of 1-hour measurements of indoor radon concentration, estimated AER is shown on the bottom part. It is well known that radon concentration in a room follows a periodical pattern, which can be explained both by meteorological parameters and by living habits (e.g. the tendency to open windows for comfortable conditions). Temporal variations in radon concentration give an opportunity to investigate the radon entry parameters and assess the dominant factors influencing these variations in more details. We suggest to use the autocorrelation function for describing the structure of the temporal dependence from time profile of radon, while the dominant periodicities in the data are examined by means of spectral analysis. Such analysis was conducted applying long-term radon time series obtained using AlphaGUARD radon monitor. 


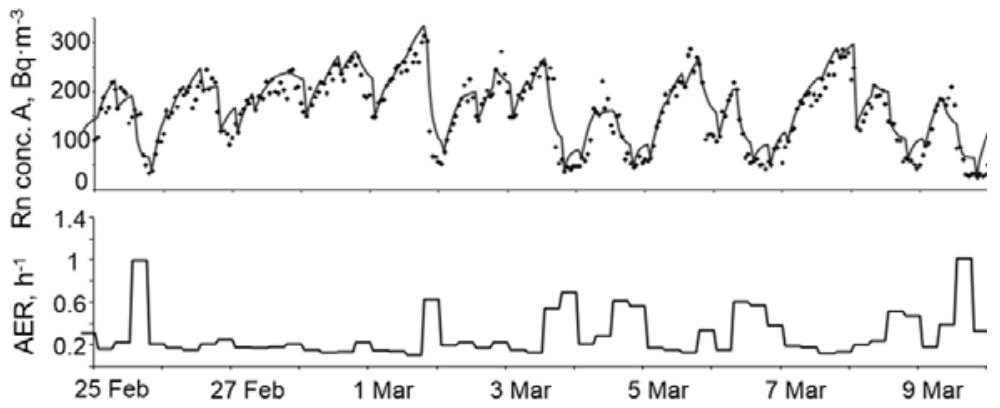

Fig. 2. Time series of 1-hour indoor radon concentration in energy-efficient building and results of preliminary analysis (on the upper part: points are results of 1-hour measurements of indoor radon concentration, curve is model; on the bottom part: estimated ventilation rate).

Software to highlight the sub-series of experimental data characterizing transition from inactive to active mode of room use was developed. Software provides automatic search of the time intervals of decreasing or increasing of indoor radon concentration. Next, the radon entry rate was obtained by the use of regression analysis [5]. Dependence of radon entry rate in a room on indoor/outdoor temperature difference allows to determine dominant mechanism of radon entry - diffusion (absence of dependence) or advective (increase of radon entry rate). Russia is country with a continental climate, typically characterized by long winter and short summer. Indoor/outdoor temperature difference attains value of 50 degrees. Finally, average contribution of the diffusion mechanism of radon entry was defined as ratio of the radon entry rate at zero degree difference between indoor and outdoor atmosphere to radon entry rate at mean temperature difference [5].

\section{Results}

Results of analysis for typical modern multi-storey buildings in Yekaterinburg, Russia are shown in the Table 1. As shown in the Table diffusion mechanism of radon entry is always dominant. Importance of the diffusion entry in modern buildings is connected with reduced air permeability and lower air exchange rate. According to the results, radon concentrations in modern multi-storey buildings in Russia are relatively high; however, the radon entry rates remain low. Therefore, the main factor leading to higher radon concentrations indoors is low ventilation rate.

Figure 3 presents Fourier spectra (raw periodograms), showing spectral density of the radon concentration as a function of period. The spectra indicates time periods that regulate the radon variations in studied dwelling the following: 12 hour, 1 day, 7 days. Autocorrelation function reveals importance of living habits in radon accumulation (Figure 4).

Table 1. Characteristic parameters of the radon entry and dilution

\begin{tabular}{|c|c|l|l|l|l|l|}
\hline ID & $\begin{array}{l}\text { Floor/number } \\
\text { of floors }\end{array}$ & $\begin{array}{l}\text { Mean radon } \\
\text { concentration, } \\
\mathrm{Bq} / \mathrm{m}^{3}\end{array}$ & $\begin{array}{l}\text { Mean air } \\
\text { exchange } \\
\text { rate, } \mathrm{h}-1\end{array}$ & $\begin{array}{l}\text { Mean } \\
\text { radon } \\
\text { entry } \\
\text { rate, } \\
\mathrm{Bq} / \mathrm{m}^{3} \mathrm{~h}- \\
1\end{array}$ & $\begin{array}{l}\text { Ra-226 } \\
\text { effective } \\
\text { concentration, } \\
\mathrm{Bq} / \mathrm{kg}\end{array}$ & $\begin{array}{l}\text { Contribution } \\
\text { of the diffusion } \\
\text { mechanism }\end{array}$ \\
\hline 1 & $7 / 16$ & 203 & $0.26 \pm 0.03$ & $38 \pm 2$ & 32 & 0.75 \\
\hline 2 & $4 / 16$ & 193 & $0.32 \pm 0.06$ & $42 \pm 3$ & 35 & 0.64 \\
\hline 3 & $3 / 24$ & 173 & $0.52 \pm 0.05$ & $63 \pm 4$ & 53 & 0.59 \\
\hline
\end{tabular}




\begin{tabular}{|c|c|c|c|c|c|c|}
\hline 4 & $6 / 10$ & 143 & $0.40 \pm 0.05$ & $33 \pm 1$ & 28 & 0.92 \\
\hline 5 & $3 / 16$ & 120 & $0.35 \pm 0.03$ & $38 \pm 2$ & 32 & 0.86 \\
\hline 6 & $12 / 16$ & 149 & $0.27 \pm 0.09$ & $31 \pm 9$ & 35 & 0.59 \\
\hline 7 & $1 / 16$ & 133 & $0.31 \pm 0.08$ & $31 \pm 7$ & 37 & 0.67 \\
\hline 8 & $15 / 25$ & 368 & $0.27 \pm 0.06$ & $65 \pm 10$ & 54 & 0.94 \\
\hline 9 & $17 / 25$ & 123 & $0.48 \pm 0.08$ & $42 \pm 5$ & 33 & 0.87 \\
\hline 10 & $6 / 16$ & 99 & $0.41 \pm 0.08$ & $38 \pm 2$ & 30 & 0.95 \\
\hline 11 & $4 / 16$ & 87 & $0.35 \pm 0.03$ & $23 \pm 2$ & 28 & 0.70 \\
\hline
\end{tabular}

As shown in the Figure 5 cumulative distribution of AER depends on the mode of room use.

It is Important to note that even the floor where room is located doesn't matter (if it is above second floor). As you can see in the Figure 6 there is no dependence on the floor for the building in which measurements were conducted on the different floors (ID 8, Table 1).

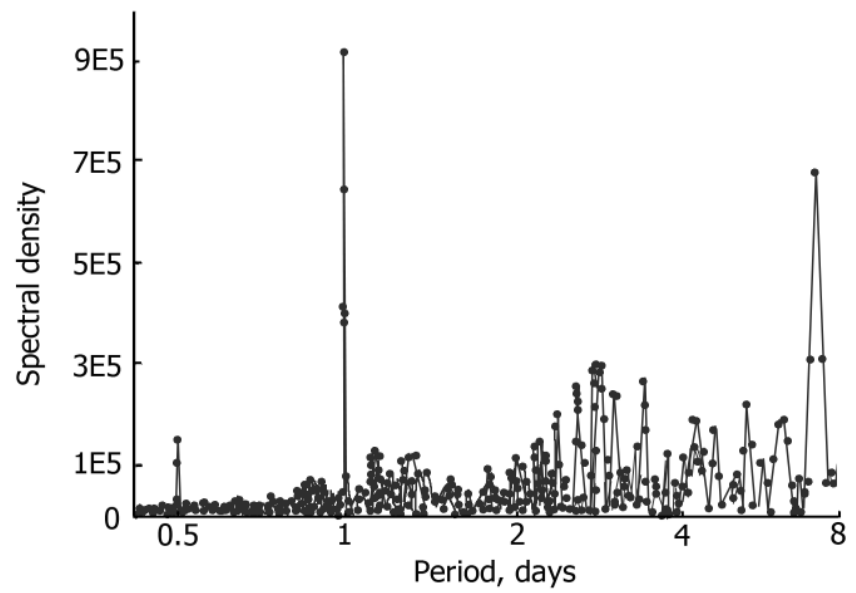

Fig. 3. Fourier spectra obtained from radon profile.

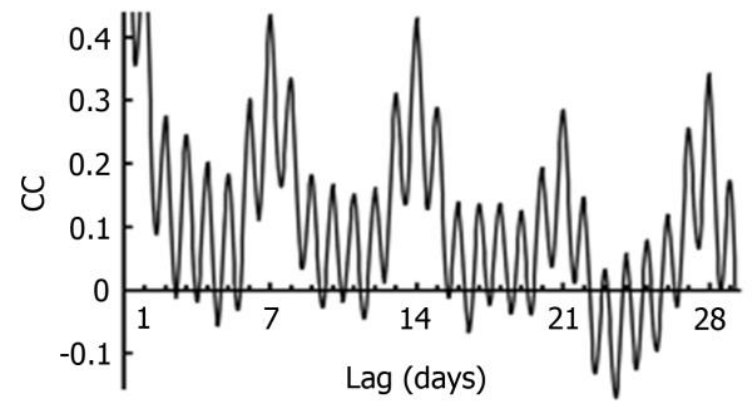

Fig. 4. Autocorrelation function obtained from radon profile. 


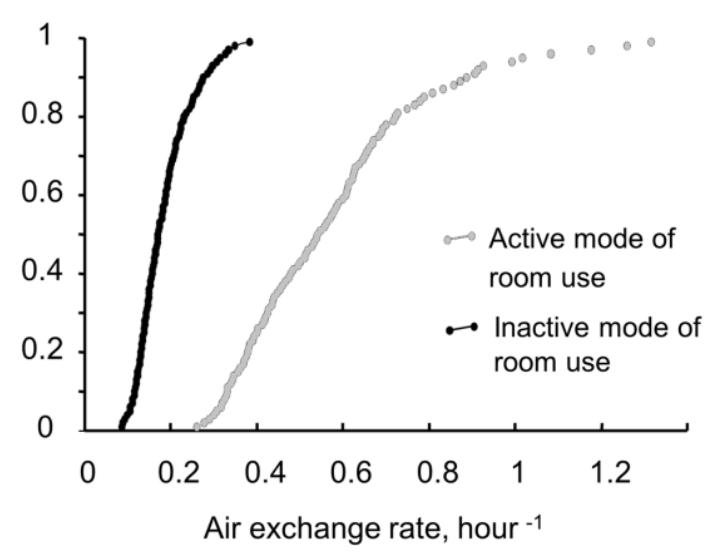

Fig. 5. Cumulative distribution of AER under active and inactive mode.

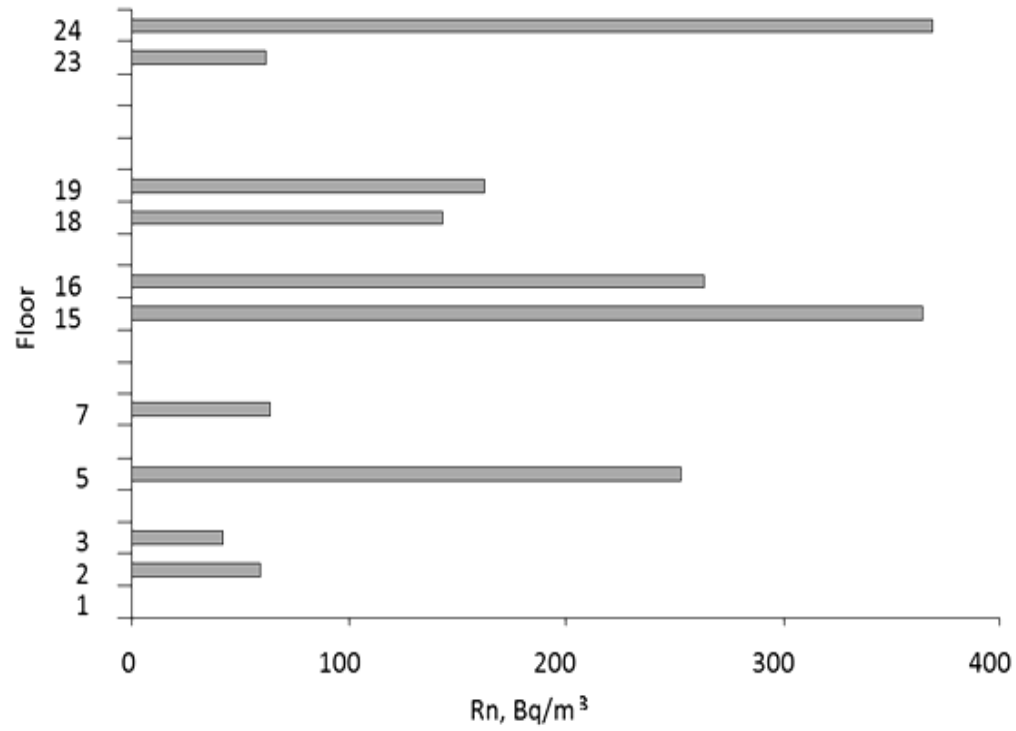

Fig. 6. Variation of radon concentration in multi-storey building (ID 8, Table 1).

\section{Conclusion}

Based on estimates of average ventilation rate under real conditions of room use in modern multi-storey dwellings, it was approved that measures to increase energy efficiency lead to reduction in the ventilation rate and high accumulation of radon concentrations indoors. Long-term time series analysis with application of mathematical methods (regression analysis, autocorrelation and spectral analysis) provides reliable and efficient technique for revealing radon entry, accumulation and dilution in energy-efficient buildings. Nevertheless, for certain purposes it is not enough yet. For example, the radon concentrations on different floors in multi-storey building are unpredictable even if we know the radon entry and dilution rates (Figure 6). It looks like one still needs to use measurements with short-term passive detectors on the various floors in multi-storey buildings. Despite the existing protocols being reasonable for single-family houses, shortterm measurements in multi-storey buildings are still an open question (number of measurements, number of floors to study and etc.) Long-term measurements with application of proposed mathematical methods give an improved understanding 
of the radon entry processes. Combined use of integrative and time series (differential) measurements provides effective way to develop standard protocol for radon measurements in energy-efficient multi-storey buildings.

\section{References}

1. J. Milner, C. Shrubsole, P. Das et al., BMJ, 348, 1-12 (2014)

2. H.R.R. Santos and M.S. Leal, Energy Build, 54, 111-121 (2012)

3. I.V. Yarmoshenko, A.V. Vasilyev, A.D. Onishchenko et al., Radiat Prot Dosim, 160, 53-55 (2014)

4. A. Vasilyev and M. Zhukovsky, J Environ Radioact, 124, 185-190 (2013)

5. A.V. Vasilyev, I.V. Yarmoshenko and M.V. Zhukovsky, Radiat Prot Dosim, 164, 601605 (2015) 